\title{
Mortality prediction for acute decompensated heart failure patient using fuzzy neural network
}

\author{
Mohamad Haider Abu Yazid a, ${ }^{*}$, Mohamad Shukor Talib a, Muhammad Haikal Satria b, Habibollah \\ Haron a zmee Abd Ghazi c \\ a School of Bioscience and Medical Engineering, Faculty of Engineering, Universiti Teknologi Malaysia, Skudai 81310, Johor, Malaysia \\ b School of Computing, Faculty of Engineering, Universiti Teknologi Malaysia, Skudai 81310, Johor, Malaysia \\ c Department of Cardiology, Institut Jantung Negara (IJN) Malaysia \\ * Corresponding author: mhaider04@gmail.com
}

\section{Article history}

Received 21 November 2019

Revised 29 January 2020

Accepted 5 February 2020

Published Online 18 August 2020

\begin{abstract}
It has been reported that patients admitted with acute decompensated heart failure (ADHF) face high risk of mortality where 30 -day mortality rates are reaching $10 \%$. Identifying patient with high and low risk of mortality could improve clinical outcomes and hospital resources allocation. This paper proposed the use of fuzzy neural network to predict mortality for the patient admitted with ADHF. Results show that fuzzy neural network can predict mortality for ADHF patient with good prediction accuracy with overall accuracy of $88.8 \%$ for partition 50 and $90.40 \%$ for partition 80 .
\end{abstract}

Keywords: mortality prediction, artificial neural network, fuzzy neural network, acute decompensated heart failure

\section{INTRODUCTION}

Acute decompensated heart failure (ADHF) is a sudden worsening of the signs and symptoms of heart failure, which typically includes difficulty breathing (dyspnea), leg or feet swelling, and fatigue. Acute decompensated heart failure is the most common cause of hospitalization for patients older than 65 years of age. Despite therapeutic advances, the prognosis of acute heart failure is poor, with in-hospital mortality ranging from $4 \%$ to $7 \%, 60$ - to 90 -day mortality ranging from $7 \%$ to $11 \%$, and 60 - to 90 -day re-hospitalization from $25 \%$ to $30 \%$. In addition, acute decompensated heart failure results in significant costs to the health care system (El-Bialy et al., 2015).

Risk of mortality varies across patient populations; a mortality prediction model (MPM) that estimates an individual patient's risk can be a useful aid for making clinical decisions at the bedside. Managing ADHF patients is challenging because of the lack of effective treatments that both reduce symptoms and improve clinical outcomes.

Lack of effective treatment that can reduce symptoms and improve clinical outcomes is one of the challenges faced by clinicians in managing ADHF patients. Knowledge of mortality predictors can be used to generate predictive model. This model can aid clinician's decision-making process especially for identifying patient who are at high or low risk of death. Risk prediction model also can be used in patient counseling to initiate the discussion of about end-of-life issues and also may be used for quality of care outcomes assessment. Patient at low risk could be potentially discharged from the hospital early and patient who identified as high risk of mortality could benefit from intensive or special care units.

Several mortality prediction models have been proposed especially for ADHF (Shao et al., 2014). However, most of the previous models use statistical/mathematical model. These models are difficult to obtain where it requires the developer of such models to understand the relationship between variables of the models. Furthermore, statistical model requires expertise and lot of effort in order to understand relationship between variables used in the model. On the other hand, machine learning techniques have become very popular to solve problems for forecasting and predictions. Machine learning works in iteration where the algorithms try to map the relationship between variables with minimal human effort. In this paper, we proposed mortality prediction framework using fuzzy neural network to predict mortality for patient admitted with ADHF.

\section{ARTIFICIAL NEURAL NETWORK MODEL}

Artificial neural network (ANN) consists of an interconnected group of artificial neurons, and it processes information using a connectionist approach to computation. ANN has been implemented in various fields. In healthcare, ANN is implemented for clinical diagnosis, drug development, image analysis, and signal analysis (Remzi et al., 2003). ANN has been proven to be useful for modeling complex relationships between inputs and outputs or to find patterns in data. Basically, feed forward neural network consists three main layers which are input layer, hidden layer and output layer. Input and output are usually consisting 1 layer and hidden layer could consist at least 1 or 2 layers. Figure 1 shows the examples of feed forward neural network architecture. The numbers of input nodes and output nodes depends on the collected data while the numbers of hidden nodes for ANN are usually based on trial and error.

ANN consists of several parameters that include number of hidden layers, number of hidden nodes, types of transfer function, and types of training algorithm. There are no specific or recommended numbers of hidden layer or hidden neuron where some researchers use trial and 
error method in order to find the best combination of parameters that form the best neural network model (Azlan Mohd Zain et al., 2010). In order to solve this problem, parameter-tuned ANN is introduced (M. Haider et al., 2018). Mortality prediction model for ADHF is developed by using previously proposed parameter-tuned ANN framework. However, the performance of mortality prediction model could still be improved by integration with fuzzy techniques.

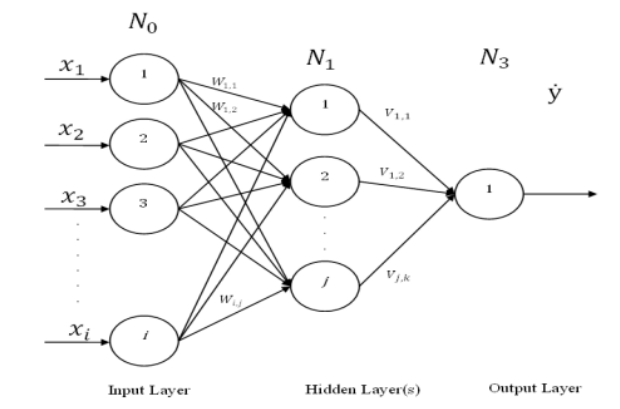

Figure 1 Feed forward neural network architecture.

Multi-layer feed forward neural network is the most frequently used algorithm in medical diagnosis. Feed forward neural network can be described in equation (2):

$$
\dot{y}_{l}=f \sum_{i} w_{i, j} x_{i}+b_{i}
$$

where $\dot{\mathrm{y}}_{j}$ is the output of the network, $f$ is the transfer function, $w_{i j}$ is the weight, $x_{i}$ is the input, and $b_{i}$ is the bias. From equation (2), multilayer feed forward neural network with 1 hidden layer can be further derived as:

$$
\dot{y}_{l}(n)=f_{k}\left(\sum_{k=1}^{N_{1}} V_{j, k} f_{j}\left(\sum_{i=1}^{N_{0}} W_{i, j} x_{i}(n)+b_{i}\right)+b_{j}\right)
$$

where, $\dot{y}$ is the output of neural network, $f$ is transfer function of the neural network, $N$ is the number of nodes in the respective layer ( $N_{0}$ the number of nodes in input layer, and $N_{l}$ is number of nodes in 1 st hidden layer), $V_{j, k}$ is the weight of neural network between a hidden layer to output layer, $W_{i, j}$ is the weight from input layer to a hidden layer, $x_{i}(n)$ is the input of neural network, and $b$ is the bias of the neural network.

The most commonly used transfer function in neural network is sigmoid function. For this research, we used sigmoid transfer function. Sigmoid transfer function (log-sigmoid) can be represented as equation (4):

$$
f=\frac{1}{1+e^{-n}}
$$

where $n$ is the output of hidden layer.

The values of weight (Wij, Vjk, Zkl) and bias (bi, bj, bk) are iteratively changed by training function in order to achieve the best prediction accuracy. Weight and bias are adjusted by training function based on the error produced by the network. This error value could be obtain using performance function. There were several performance functions that can be used in neural network. We used MSE (Mean Squared Error) and Cross Entropy performance function in this research. For multi class classification problems, cross entropy is widely used as neural network performance function. However, some of the training function use Jacobian-based matrix calculation (e.g. Lavenberg-Marquadt and Bayesian Regularization) to find MSE or SSE (Sum Squared Error) for weight and bias calculation. Cross
Entropy produces error values that heavily penalizes outputs that are extremely inaccurate $(\dot{y}$ near $1-t)$, with very little penalty to fairly correct classification/prediction ( $\dot{y}$ near $\mathrm{t}$ ). Minimizing cross entropy and MSE valued leads to good neural network model. Cross Entropy using equation (5) to produced error values and MSE using equation (6):

$$
\begin{aligned}
& \text { error }=E=-t_{l} \cdot \log \dot{y}_{l} \\
& \text { error }=E=\frac{1}{2}\left(t_{l}-\dot{\mathrm{y}}_{l}\right)^{2}
\end{aligned}
$$

where $t_{l}$ is the output target and $\dot{y}_{l}$ are the neural network predicted output. After the error value is compute, the training algorithm will adjust the weight and bias of the neural network based on the error value. The training functions that often used by researcher in field of medical diagnosis are back propagation algorithm. Back propagation algorithm is a learning function and commonly method for training neural networks where it used gradient descent algorithms that minimize squared error. Squared errors are minimized by using iterative process of gradient descent. Gradient descent can be expressed in equation (7):

$$
g_{i}=\frac{\partial E_{i}}{\partial W_{i}}
$$

where $g_{i}$ the gradient of ith iteration, $E$ is the error of the network for the $i$ th iteration, and $W$ the weight and biases of $i$ th iteration. Weight and biases are updated in the direction of network error (performance function) decreases most rapidly (negative of gradient) using equation (8):

$$
U_{i+1}=U_{i}-\eta g_{i}
$$

where $U_{i}$ a vector of current weight and biases, $g_{i}$ is the current gradient and $\eta$ is the learning rate (proportional parameter which defines the step length of each iteration in the negative gradient descent). Learning rate value is defined by the user where the small value of $\eta$ could lead to true approximation or prediction while slowing learning process. However, choosing larger value of $\eta$ could speed up neural network convergence while may cause oscillation in weight spaces. Basically, back propagation works as follows:

1) Neural network is given input $\mathrm{x}$ and error of the network is calculated,

2) Sensitivities $\left(\Delta W_{i}\right.$ and $\left.\Delta b_{i}\right)$ are propagated from output layer to the first layer and

3) The weight $w$ and biases $b$ of the neural network is updated.

Back propagation uses the chain rule in order to compute derivatives of the squared error with respect to the weights and biases in the hidden layers.

This algorithm called back propagation because the derivatives are computed first at the last layer of the network and then propagated backward through the network to compute the derivatives in the hidden layer.

\section{PROPOSED FUZZY NEURAL NETWORK FRAMEWORK}

This paper proposed a framework for mortality prediction for ADHF patient using fuzzy neural network. Figure 2 illustrates the proposed fuzzy neural network framework for mortality prediction for ADHF patient. Data used for this research were collected from 
Cardiology Medical Record (ADHF) Database in National Heart Institute (IJN) Malaysia. Ethical approval from IJN Ethics Committee was obtained before data collected process was conducted. Data collected in this research consist of patient from period of 1st January 2009 until 22nd December 2015. From the database, 60 inputs attributes of the neural networks that include patient profile (gender, age, weight etc.), patient medical history (history of PCI procedure, CABG procedure, Myocardial Infarction, etc.), patient medical diagnosis (patient blood pressure, urea level, sodium level, etc.), patient hospital procedure (dialysis, angiography, etc.) have been extracted. Patient status during hospitalization (deceased or alive) was used as target of the neural network. Table 1 shows the input and output used for mortality prediction framework for ADHF patients.

Table 1 Input and Output of proposed variance prediction using ANN.

\begin{tabular}{|c|c|c|}
\hline \multicolumn{2}{|c|}{ INPUT } & \multirow[b]{2}{*}{$\begin{array}{l}\text { TARGET } \\
\text { /OUTPUT }\end{array}$} \\
\hline $\begin{array}{l}\text { Patient Medical History } \\
\text { Record and Current } \\
\text { Medical Condition }\end{array}$ & $\begin{array}{l}\text { Patient's Medical } \\
\text { Diagnosis }\end{array}$ & \\
\hline $\begin{array}{l}\text { 1. Gender } \\
\text { 2. Age } \\
\text { 3. Weight } \\
\text { 4. Height } \\
\text { 5. Smoking Habit } \\
\text { 6. Previous Heart } \\
\text { Failure } \\
\text { Hospitalization } \\
\text { 7. Coronary Artery } \\
\text { Disease } \\
\text { 8. Previous PCI } \\
\text { Procedure } \\
\text { 9. Previous CABG } \\
\text { Procedure } \\
\text { 10. Previous MI } \\
\text { 11. Renal Insufficiency } \\
\text { 12. Creatinine more than } \\
\text { 37 } \\
\text { 13. Regular Dialysis } \\
\text { 14. Atrial Fibrillation } \\
\text { 15. Diabetes } \\
\text { 16. Hypertension } \\
\text { 17. Hyperlipidemia/Dysli } \\
\text { pidemia } \\
\text { 18. Stroke } \\
\text { 19. COPD/Asthma } \\
\text { 20. Dyspnea } \\
\text { 21. Peripheral Edema } \\
\text { 22. Ascites } \\
\text { 23. Lung Crepitation } \\
\text { 24. Elevated JVP } \\
\text { 25. Hepatomegaly } \\
\text { 26. ECG Procedure } \\
\text { Done } \\
\text { 27. Rhythm ID } \\
\text { 28. Q Wave } \\
\text { 29. ST Segment } \\
\text { Depression } \\
\text { 30. ST Segment } \\
\text { Elevation } \\
\text { 31. No Infarction } \\
\text { Evidence } \\
\text { 32. X-ray Procedure } \\
\text { 33. Cardiomegaly } \\
\text { 34. Pleural Effusion } \\
\text { 35. Congestion } \\
\text { 36. Ill-defined Opacity } \\
\text { 37. Acute Pulmonary } \\
\text { Edema } \\
\text { 38. LVEF not done } \\
\text { 39. Dialysis Procedure } \\
\text { 40. PCI Procedure } \\
\text { 41. Angiography } \\
\text { Procedure } \\
\text { 42. Cardiac Catheter } \\
\text { Procedure } \\
\text { 43. MRI/CT Scan } \\
\text { Procedure } \\
\text { 19. }\end{array}$ & $\begin{array}{l}\text { 44. QRS Duration } \\
\text { 45. Systolic Blood } \\
\text { Pressure } \\
\text { 46. Diastolic Blood } \\
\text { Pressure } \\
\text { 47. Heart Rate } \\
\text { 48. Respiratory } \\
\text { Rate } \\
\text { 49. Pre- } \\
\text { Hospitalization } \\
\text { Left } \\
\text { Ventricular } \\
\text { Ejection } \\
\text { Fraction } \\
\text { (LVEF) } \\
\text { 50. Left } \\
\text { Ventricular } \\
\text { Ejection } \\
\text { Fraction } \\
\text { (LVEF) during } \\
\text { admission. } \\
\text { 51. Urea level } \\
\text { 52. Sodium level } \\
\text { 53. Potassium } \\
\text { level } \\
\text { 54. Creatinine } \\
\text { level } \\
\text { 55. Uric Acid level } \\
\text { 56. Random Blood } \\
\text { Sugar level } \\
\text { 57. Bilirubin level } \\
\text { 58. Albumin level } \\
\text { 59. Creatine } \\
\text { Kinase (CK) } \\
\text { level } \\
\text { 60. Hemoglobin } \\
\text { level }\end{array}$ & $\begin{array}{c}\text { In } \\
\text { Hospitalization } \\
\text { Death }\end{array}$ \\
\hline
\end{tabular}

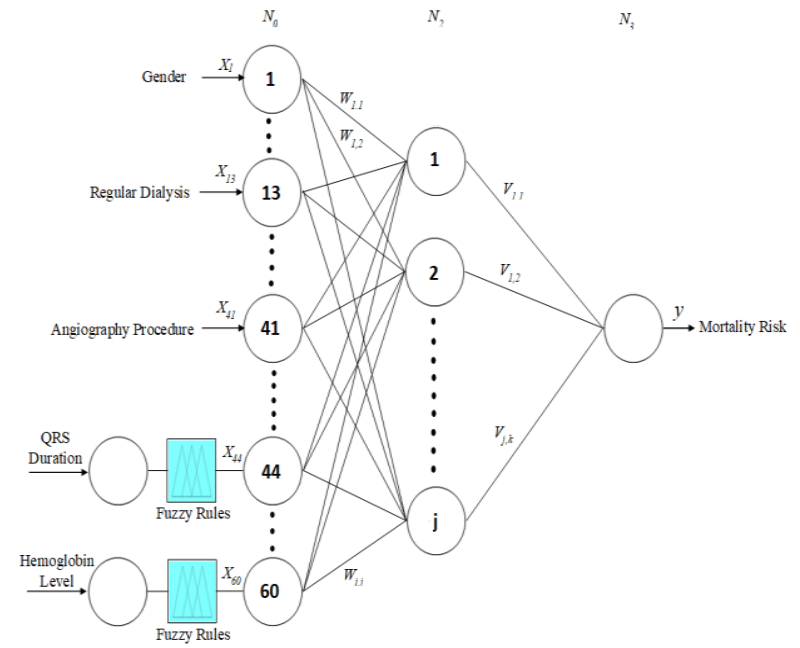

Figure 2 The proposed fuzzy neural network for mortality prediction.

The proposed fuzzy neural network utilized fuzzy logic method to transform patient's medical diagnosis data into fuzzified values. The patient's medical diagnosis data contains non-linear and imprecise data. This imprecision could be illustrated by taking examples of interpretation of albumin level in blood test. Albumin blood test is used to measures the condition of patient's liver function. High or low level of albumin in blood may indicates medical problems (Sridevi Devaraj, 2015).

Figure 3 shows the interpretation of albumin abnormality range where 1 is considered abnormal value while 0 indicates normal value. The graph shows that low and very high level of albumin is considered abnormal value. It shows the non-linear nature of albumin level. Furthermore, the interpretation level may slightly vary in different laboratories (Sridevi Devaraj, 2015). These variations can lead to imprecision in data.

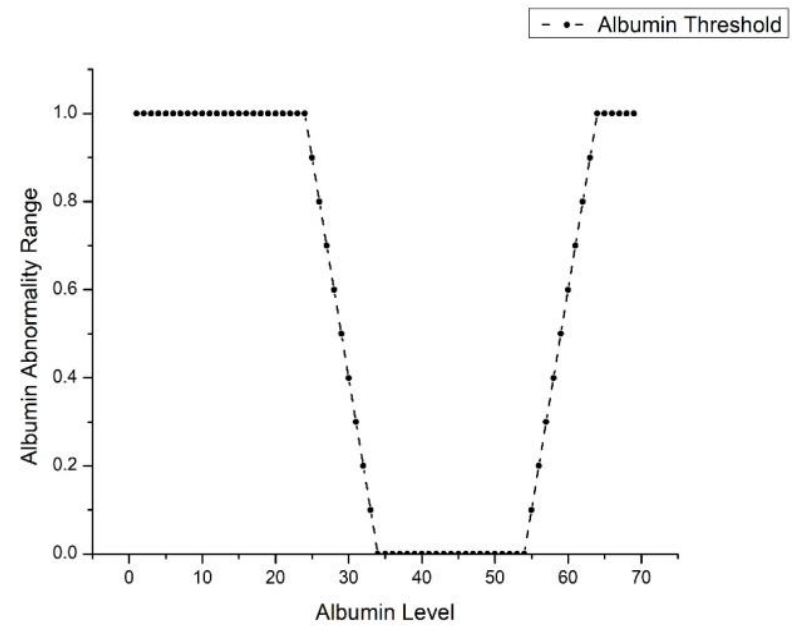

Figure 3 The Interpretation of albumin level in blood.

Non-linearity and imprecision in data could affect the performance of the proposed mortality prediction model. Several researchers such as Paolo Giordani (2010) and Robert Niwicki et al. (2009) have proposed the use of fuzzy logic to resolve the aforementioned problems.

\section{FUZZIFICATION}

\section{Overview}

There are three basic processes in fuzzy logic which are:

1. Input fuzzification into fuzzy membership function 
2. Implementation of all relevant rules in rule base to compute the fuzzy output functions.

3. Fuzzy output defuzzification for obtaining "crisp" output values

Based on these three processes, there are three main components to the data fuzzification of Acute Decompensated Heart Failure dataset. These three main components are:

1. Rule base of each attributes.

2. Input membership function.

3. Output membership function.

Rule base of each attributes are the interpretation rules of medical diagnosis or lab results for each patient in ADHF dataset. For example, bilirubin level in blood is considered high when its level is higher than $32 \mu \mathrm{mol} / \mathrm{L}$. This interpretation or rules will determine input membership function of each attributes. Output membership function is determined by the "severity' of the medical diagnosis or lab results of the patients. For example, bilirubin blood test is often used to diagnose liver diseases in patient. Normal level does not indicate any medical issues while high level of bilirubin may indicate several medical conditions such as hepatitis or liver dysfunction. Based on this information, the high level of bilirubin will be categorized as severe and normal level of bilirubin is categorized as normal in output membership function.

However, most of the medical diagnosis or lab results is not as straightforward as the bilirubin examples. There are several attributes that have "normal" values between the observable range. For examples, the albumin level is considered normal when the level between 35 to 55 $\mathrm{g} / \mathrm{L}$ while it considered as low when the level is below 34 and high when the level is higher than $55 \mathrm{~g} / \mathrm{L}$. The severity of condition is also different between each attribute where the low level may indicate severe conditions compared to high level.

After the information regarding rule base and interpretation rules is obtained, the membership function for input and output is created. In this research, trapezoidal membership function is used. Trapezoidal membership function is used in this research because it is suitable for all the attributes that need to be fuzzified in ADHF. These attributes have definitive range that associated with their respective level. For examples, the albumin level is considered normal when its level in blood is between 35 to $55 \mathrm{~g} / \mathrm{L}$. Any reading that out of this range is considered abnormal whether the albumin level is low or high. So, in this case, if the level of albumin is between 40 to $50 \mathrm{~g} / \mathrm{L}$, it definitely belongs to the normal level membership function. Triangle, Gaussian, and Sigmoid membership are not suitable for this kind of attributes.

\section{Fuzzification Examples}

Fuzzification is applied to the 16 attributes in this research. Every attribute have different rules, input membership function and output membership function. Because of the limitation, only albumin blood test fuzzification as an example for this paper. Albumin blood test are used to evaluate liver function. The low level of albumin in the blood may indicate:
1. Liver Disease
2. Kidney Disease
3. Malnutrition
4. Infection
5. Inflammatory bowel disease
6. Thyroid Disease

Higher level of albumin may indicate severe dehydration or severe diarrhea. Patient will have increase mortality rate when albumin level is low because it indicates severe medical condition as listed above. The levels of albumin values maybe vary between laboratories. Table 2 shows the interpretation of Albumin blood test levels according to the sources obtain from literature review (Sridevi Devaraj, 2015).
Table 2 Albumin level interpretation.

\begin{tabular}{cc}
\hline Level & Values \\
\hline Low & $<34 \mathrm{~g} / \mathrm{L}$ \\
\hline Normal & $35-55 \mathrm{~g} / \mathrm{L}$ \\
\hline High & $>55 \mathrm{~g} / \mathrm{L}$
\end{tabular}

Fuzzy membership function and fuzzy rules for Albumin level is created based on Table 2 and Figure 3 which show the input membership function for albumin blood test levels. As mentioned above, albumin blood test is used to evaluate liver function where low levels of albumin in blood may indicate deterioration of liver function while high level albumin may be due to several factors such as dehydration but not necessarily indicate deterioration of liver function. Trapezoidal membership function is used in this research. Table 3 shows the range of input membership function and Table 4 shows the range of output membership function used in this research

Table 3 Input Membership function range.

\begin{tabular}{|c|c|}
\hline $\begin{array}{c}\text { Input Membership } \\
\text { function }\end{array}$ & Range \\
\hline Low & {$\left[\begin{array}{lllll}0 & 0 & 30 & 35\end{array}\right]$} \\
\hline Normal & [30 355560$]$ \\
\hline High & {$\left[\begin{array}{lllll}50 & 60 & \infty\end{array}\right]$} \\
\hline
\end{tabular}

Table 4 Output Membership function range.

\begin{tabular}{cc}
\hline $\begin{array}{c}\text { Output Membership } \\
\text { function }\end{array}$ & Range \\
\hline Normal & {$\left[\begin{array}{llll}0 & 0 & 0.4 & 0.5\end{array}\right]$} \\
\hline Moderate & {$\left[\begin{array}{llll}0.4 & 0.5 & 0.6 & 0.7\end{array}\right]$} \\
\hline Severe & {$\left[\begin{array}{llll}0.6 & 0.7 & 1 & 1\end{array}\right]$} \\
\hline
\end{tabular}

The output membership function for albumin blood test is created using this information and represented in Figure 4.

Fuzzy rules are created after fuzzy membership function is specified. The rules for fuzzified albumin blood test are as follow:

1. If (Albumin Blood Test Level) is Low, then (Albumin Fuzzified Value) is Severe

2. If (Albumin Blood Test Level) is Normal, then (Albumin Fuzzified Value) is Normal

3. If (Albumin Blood Test Level) is High, then (Albumin Fuzzified Value) is Moderate

By using the fuzzification techniques, patient's albumin blood level is transformed to severity scale. For examples, patient's with albumin level of 46 (normal levels) is transformed to values close to 0 in term of severity which indicates not severe while patients which albumin level of 20 (low) is transformed to values close to 1 which indicates that the condition is severe. The severity levels of patients with slight elevation of albumin (more than 55) level will produce values between 0.4 until 0.7 .

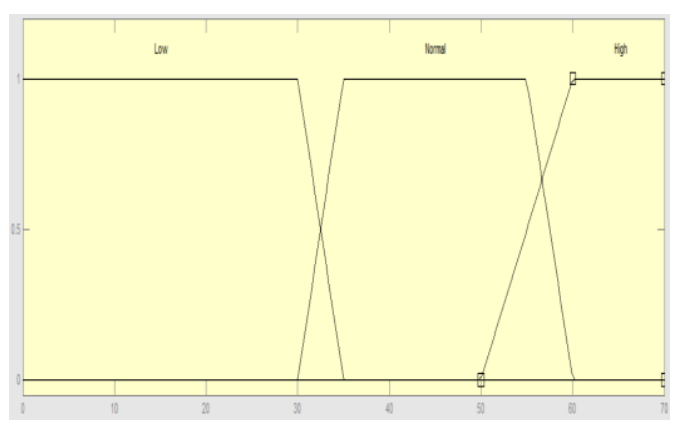


Figure 3 Albumin Blood Test Level membership function for input.

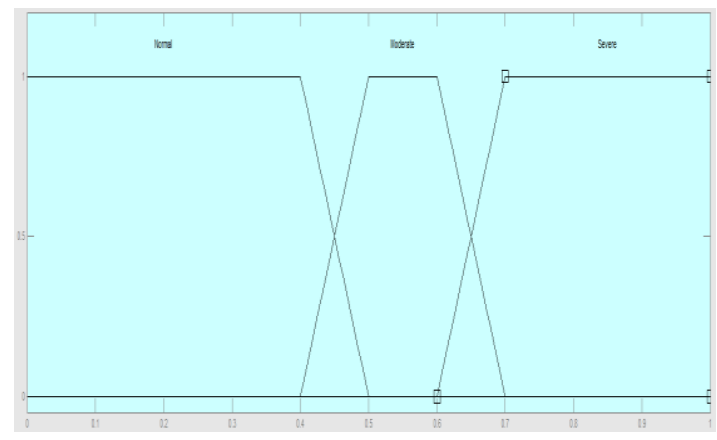

Figure 4 Albumin Blood Test Level membership function for output.

\section{EXPERIMENTAL SETUP}

In this research, ADHF dataset is classified into two sets. The first dataset is grouped by ratio of 50:25:25 where 50\% of dataset is used for training and another $25 \%$ is used for validation and testing. The second dataset consist of training, validation, and test ration of 80:10:10. The first dataset of 50:25:25 ratios is to simulate the dataset with low ratio of training dataset while the second dataset of 80:10:10 ratio is chosen to simulate the dataset with significant amount of training data. Using this method, this research could evaluate which classification techniques that able to produce good accuracy in situation where the training data is lacking and where the training data is sufficient.

The proposed framework is simulated using MATLAB R2017b on the computer with following specifications: Intel Core i-7 with 2.60 GHz, RAM 12.0 GB, and 64-bit processing system. The proposed fuzzy neural network for ADHF Mortality prediction utilizes parameter tuning framework proposed by M. Haider et al (2018) to obtained the optimized neural network parameters. Table 5 shows the parameters obtained using parameter tuning framework for the proposed fuzzy neural network for ADHF Mortality Prediction.

Table 5 Neural network parameter for fuzzy neural network.

\begin{tabular}{cccccc}
\hline \multicolumn{5}{c}{ Neural Network Parameters } \\
\hline $\begin{array}{c}\text { Neural } \\
\text { Motworks }\end{array}$ & $\begin{array}{c}\text { Training } \\
\text { Algorithm }\end{array}$ & $\begin{array}{c}\text { Hidden } \\
\text { Layer }\end{array}$ & $\begin{array}{c}\text { Hidden } \\
\text { Nodes } \\
\text { Layer } \\
\text { Transfer } \\
\text { Function }\end{array}$ & $\begin{array}{c}\text { Output } \\
\text { Layer } \\
\text { Transfer } \\
\text { Function }\end{array}$ \\
\hline $\begin{array}{c}\text { Fuzzy } \\
\text { Neural } \\
\text { Network }\end{array}$ & $\begin{array}{c}\text { Scaled } \\
\text { conjugate }\end{array}$ & 1 & 60 & $\begin{array}{c}\text { Satlin } \\
\text { (Saturating } \\
\text { Linear) }\end{array}$ & $\begin{array}{c}\text { Satlins } \\
\text { (Symmetric } \\
\text { Saturating } \\
\text { Linear) }\end{array}$ \\
\hline
\end{tabular}

After the results for the proposed fuzzy neural network was obtain, the simulation proceeds by using existing machine learning algorithm. The overall results were analyzed and the accuracy of each algorithms was compared.

\section{RESULTS AND DISCUSSION}

Table 6 shows the overall classification results obtain by the proposed framework compared with existing machine learning algorithm for partition 50 and Figure 5 shows the graph of the prediction accuracy shown in table 6. Prediction accuracy for partition 80 is shown in Table 7 and its graph is shown in Figure 6.

For partition 50, the proposed fuzzy neural network able to achieve good prediction accuracy compared to the others existing machine learning techniques. Overall accuracy obtain by the proposed fuzzy neural network is $88.8 \%$ percent while test dataset accuracy obtain is $86.7 \%$ of accuracy.
For partition 80 , the proposed fuzzy neural network also able to achieve good prediction accuracy. The highest overall accuracy obtains for partition 80 is obtain by random tree algorithm and $\mathrm{k}$ nearest neighbors algorithm. However, the accuracy dataset for both of these algorithms is low compared to the others algorithms. It shows that these algorithms suffer from overfitting problems where the training data achieved high accuracy while the accuracy of test dataset is low. The proposed fuzzy neural network able to achieve highest accuracy for test dataset and also obtain good overall accuracy.

Table 6 Prediction accuracy comparison for partition 50.

\begin{tabular}{ccccc}
\hline \multicolumn{5}{c}{ Partition 50} \\
\hline Algorithm & Training & Validation & Test & Overall \\
\hline Naïve Bayes & 87.5 & 80 & 76.66 & 82.7 \\
\hline Random Tree & 100 & 71.28 & 58.38 & 82.5 \\
\hline SVM & 100 & 55.45 & 43.33 & 74.58 \\
\hline $\begin{array}{c}\text { Adaptive } \\
\text { Boosting }\end{array}$ & 84.16 & 75 & 78.21 & 80.41 \\
\hline CART & 82.5 & 81.66 & 76.66 & 80.83 \\
\hline $\begin{array}{c}\text { Linear Logistic } \\
\text { Regression }\end{array}$ & 87.50 & 85.00 & 80.00 & 85.00 \\
\hline kNN & 100.00 & 70.00 & 66.67 & 84.50 \\
\hline Neural Network & 85 & 85 & 78.3 & 83.3 \\
\hline $\begin{array}{c}\text { Proposed } \\
\text { Fuzzy Neural } \\
\text { Network }\end{array}$ & 89.2 & 90 & 86.7 & 88.8 \\
\hline
\end{tabular}

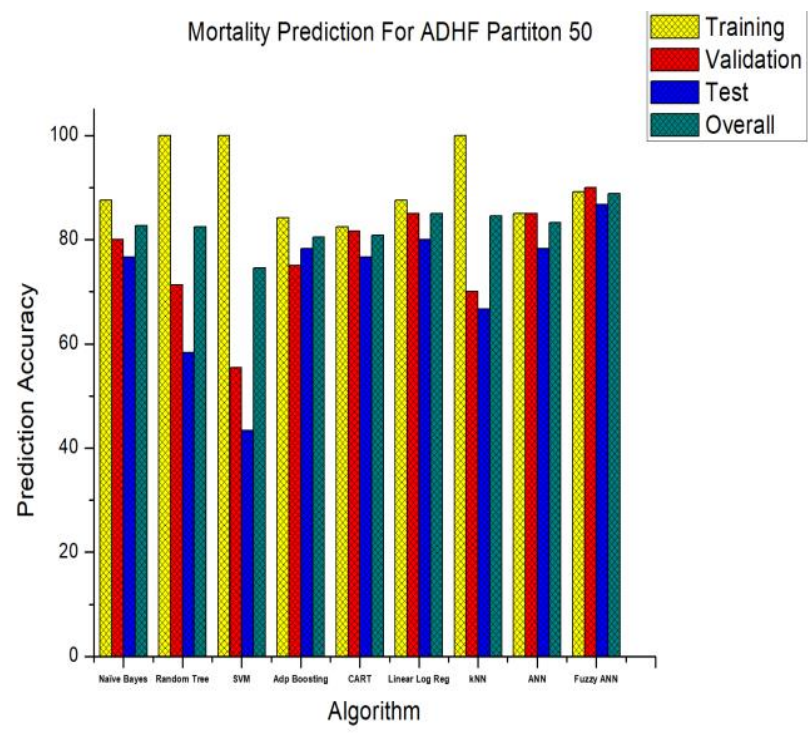

Figure 5 Prediction Accuracy for Partition 50

Table 7 Prediction accuracy comparison for partition 80 .

\begin{tabular}{ccccc}
\hline \multicolumn{5}{c}{ Partition 80 } \\
\hline Algorithm & Training & Validation & Test & Overall \\
\hline Naïve Bayes & 83.16 & 68.00 & 76.00 & 81.25 \\
\hline Random Tree & 100.00 & 80.00 & 52.00 & 92.91 \\
\hline SVM & 100.00 & 44.00 & 40.00 & 87.91 \\
\hline Adaptive Boosting & 83.16 & 76.00 & 84.00 & 82.90 \\
\hline CART & 82.10 & 72.00 & 80.00 & 80.83 \\
\hline $\begin{array}{c}\text { Linear Logistic } \\
\text { Regression }\end{array}$ & 88.94 & 72.00 & 88.00 & 87.08 \\
\hline kNN & 100.00 & 52.00 & 72.00 & 92.00 \\
\hline Neural Network & 90.10 & 87.50 & 91.70 & 90.00 \\
\hline $\begin{array}{c}\text { Proposed Fuzzy } \\
\text { Neural Network }\end{array}$ & 90.60 & $\mathbf{8 3 . 3 0}$ & 95.80 & 90.40 \\
\hline
\end{tabular}




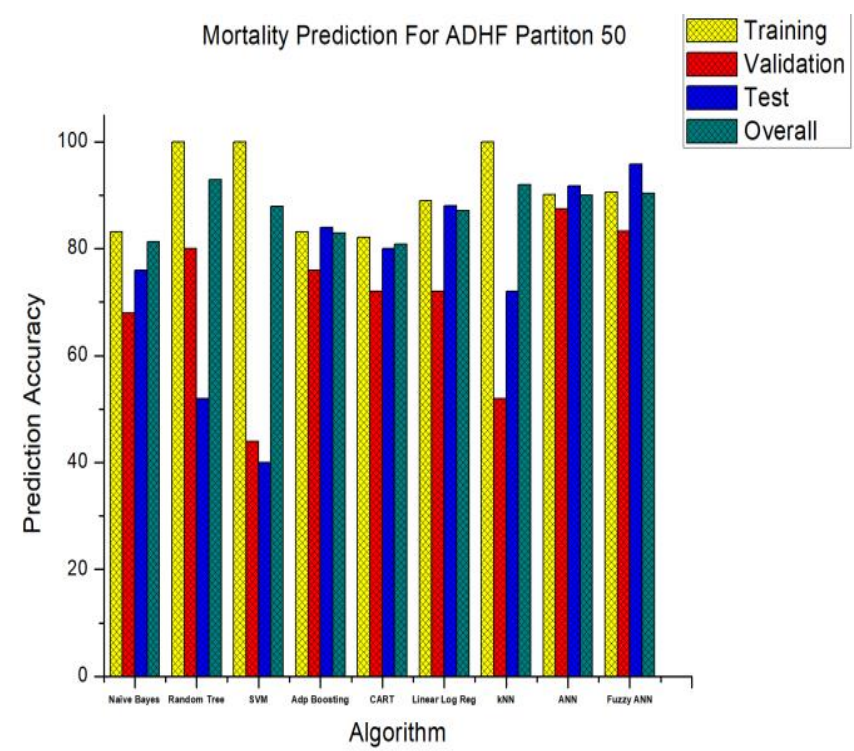

Figure 6 Prediction accuracy for partition 80.

\section{CONCLUSION}

This paper proposed the fuzzy neural network framework for ADHF mortality prediction. Based on the simulation results and comparison with others existing algorithms, several conclusions could be made as follows:

1. The proposed fuzzy neural network algorithm is able to achieve good prediction accuracy for ADHF mortality predictions.

2. The implementation of fuzzy with neural network able to increase the performance of standard neural network especially for the model that contains less data (as simulated in partition 50).

3. Several existing algorithms tend to over fit the prediction model where the prediction accuracy of training dataset is high but the prediction accuracy of test dataset is low.

\section{ACKNOWLEDGEMENT}

This work was financially supported by the Universiti Teknologi Malaysia under the Research University Grant and Ministry of Higher Education Malaysia.

\section{REFERENCES}

Abu Yazid, M. H., Talib, S., Satria, M. H. 2018. Artificial neural network parameter tuning framework for heart disease classification. Proceeding of the Electrical Engineering Computer Science and Informatics, 5: 674-679.

Anooj, P. K. 2012. Clinical decision support system: Risk level prediction of heart disease using weighted fuzzy rules. Journal of King Saud UniversityComputer and Information Sciences 24(1): 27-40.

Blake, C. L., Merz, C. J. UCI Repository of machine learning databases. Irvine, C. A. 1998. http://www.ics.uci.edu/ mlearn/MLRepository.html

Das, R., Turkoglu, I., Sengur, A. 2009. Effective diagnosis of heart disease through neural networks ensembles. Expert Systems with Applications, 36(4):7675-7680.

Devaraj, S. 2015. Drugs \& Diseases, Laboratories Medicine, Albumin. Available from: https://emedicine.medscape.com/article/2054430overview>.

El-Bialy, R., Salamay, M. A., Karam, O. H., Khalifa, M. E. 2015. Feature analysis of coronary artery heart disease data sets. Procedia Computer Science, 65:459-468.

Hedeshi, N., Ghadiri, Abadeh. M. S. 2014. Coronary artery disease detection using a fuzzy-boosting PSO approach. Computational Intelligence and Neuroscience, 6: 1-12.

Shao, Y. E., Hou, C.-D., Chiu, C.-C. 2014. Hybrid intelligent modeling schemes for heart disease classification. Applied Soft Computing Journal, 14: 47-52.

Nahato, Biredagn, N., Harichandran, K. N., Arputharaj, K. 2015. Knowledge mining from clinical datasets using rough sets and backpropagation neural network. Computational and Mathematical Methods in Medicine.

Nowicki, Robert K., Janusz, T. Starczewski. 2017. A new method for classification of imprecise data using fuzzy rough fuzzification. Information Sciences, 414: 33-52.

Paolo, G. Three-way analysis of imprecise data. 2010. Journal of Multivariate Analysis, 101(3), 568-582.

Polat, K., Sahan, S., Kodaz, H., Günes, S. 2005. A new classification method to diagnosis heart disease: Supervised artificial immune system (AIRS). Proceedings of the Turkish Symposium on Artificial Intelligence and Neural Networks (TAINN).

Remzi, M., Anagnostou, T., Ravery, V., Zlotta, A., Stephan, C., Marberger, M., Djavan, B. 2003. An artificial neural network to predict the outcome of repeat prostate biopsies. Urology, Sep; 62(3):456-460.

Sagir, Masanawa, A., Sathasivam, S. 2017. A novel adaptive neuro fuzzy inference system based classification model for heart disease prediction. Pertanika Journal of Science \& Technology 25(1).

Vijaya, K., Nehemiah, H. K., Kannan, A, Bhuvaneswari, N.G. 2010. Fuzzy neuro genetic approach for predicting the risk of cardiovascular diseases. International Journal of Data Mining, Modelling and Management, 2(4): 388-402.

Xu, Y., Pan, X., Zhou, Z., Yang, Z., Zhang, Y. 2015. Structural least square twin support vector machine for classification. Applied Intelligence, 42(3):527536.

Zain, Mohd, A., Haron, H., Sharif, S. 2010. Prediction of surface roughness in the end milling machining using Artificial Neural Network. Expert Systems with Applications 37(2): 1755-1768.

Zhang, Guoqiang, B., Patuwo, E., Michael, Y. H. 1998. Forecasting with artificial neural networks: The state of the art. International Journal of Forecasting 14(1): 35-62. 